\title{
Überlegungen zum pädagogischen Fachbegriff „Literacy“ für das Deutschlernen in Taiwan'
}

\author{
Shu-Ping Lee \\ Asia University \& Fu-Jen University \\ leeshuping.phd@gmail.com \\ https://dx.doi.org/10.12795/futhark.2013.i08.08
}

\begin{abstract}
This project aims at exploring the concept of "literacy" in the context of German learning in Taiwan in order to clarify the significance of how the competence of critical cross-cultural communication to be embedded in the curriculum. Literacy is a term derives from the American context. The German context tends to regard it as a pedagogic term for reading, narrating and writing culture. In the context of traditional four-skill language training (reading, listening, writing and speaking), "literacy" implies not only the necessity of acquiring various contents including political, economic, social issues or the advance in science, but also the ability of conveying abstract ideas. In this sense, literacy refers to what Gee (1991) defines as secondary discourses, for which a socio-cultural perspective can be employed in a content-oriented and genre-based curriculum where social practice is emphasized. This curriculum leads to the possibility that language could be treated as culture (Kramsch 1995) and a third place (Kramsch 2009) could hence be critically created between the self and the other with the redefined concept of literacy for repositioning the identity of German learners in Taiwan.
\end{abstract}

Key words: literacy, intercultural understanding, German learning in Taiwan, the socio-cultural perspective, a third space

Abstract: Die vorliegende Arbeit diskutiert "Literacy“ im Kontext des Deutschlernens in Taiwan, um zu erklären, wie die Kompetenz einer kritischen interkulturellen Kommunikation erzielt werden kann. Aus dem AngloAmerikanischen stammend und im Deutschen als pädagogischer Fach- und Sammelbegriff für Lese-, Erzähl- und Schrift- kultur geltend, werden dabei im Vergleich zum herkömmlichen Deutschlernen (Lese-, Hör-, Schreib- und

Diese Arbeit ist eine revidierte Version des Vortragstexts, den die Authorin im internationalen Rikkyo-Kolloquium 2011 in Tokyo, Japan, präsentiert hat, und wurde nach der Tagung anhand der Kommentare neu bearbeitet. 
Sprech- Verständnis) vor allem die aktive Politik, aktuelle Wirtschaft, pulsierende Gesellschaft und der neueste Stand der Wissenschaft, oder gar die hohe Kunst der sprachlichen Abstraktionsfähigkeit vermittelt. Literacy ist in diesem Sinne nach Gee (1991) mit den Sekundärdiskursen identisch, die im Deutschcurriculum durch inhaltlich- orientierte und Genre-basierende Handlungen mit einer soziokulturellen Perspektive vermittelt werden. Eine geradezu perfekte Möglichkeit, die Sprache als Kultur (Kramsch 1995)im dritten Bereich (Kramsch 2009) zwischen dem Eigenen und dem Fremden kritisch interkulturell zu betrachten, und eine Verpflichtung, die Identität des Deutschlernens in Taiwan anhand von Literacy neu zu durchdenken.

Leitbegriffe: Literacy, interkulturelles Verstehen, Deutschlernen in Taiwan, die soziokulturelle Perspektive, ein dritter Bereich

\section{Einleitung}

„Literacy“ stammt aus dem Anglo-Amerikanischen und gilt im Deutschen als pädagogischer Fach- und Sammelbegriff für Lese-, Erzähl- und Schrift- kultur. In dieser vorliegenden Arbeit wird schwerpunktmäßig auf das Hauptthema "Literacy“ als Begriff eingegangen. Es wird aufgezeigt, welche Rolle „Kultur" im Deutschunterricht Taiwans spielen kann, worin die potentielle Problematik der „interkulturellen Kommunikation“ besteht und wie bestehende Barrieren des Deutschunterrichts auf systemimmanente Weise überwunden werden. Davon ausgehend bediene ich mich einer soziokulturellen Perspektive, um das gegenseitige Verstehen der Kulturen durch die Konzepte „languaculture“ (Lantolf 2006) bzw. „language as culture"(Kramsch 1995) wahrzunehmen.

In Taiwan gibt es 171 Hochschulen (inkludiert sind Universitäten, Technische Universitäten und Colleges), darunter aber nur 8 Universitäten mit Deutschabteilungen bzw. Deutschprogrammen, und von diesen haben nur 3 Masterprogramme mit Schwerpunkten von Sprachwissenschaft, Literatur, interkulturelle Kommunikation, Didaktik und Dolmetschen/Übersetzen. Die Bemühungen des Deutschlernens in Taiwangelten dem Ziel, Deutsch nicht nur in Lehre und Forschung zu etablieren, sondern auch das Studium durch Fremdsprachenlernen zu internationalisieren.

Wegen der schnellen Entwicklung von Technologie und Kommunikation so wieder daraus folgenden Globalisierung, befinden wir uns jetzt in einer Zeit der sehr raschen Veränderungen. Viele Medien sind für die Lernenden einfach erreichbar und innerhalb oder außerhalb des Unterrichts benutzbar. Womit sie einen authentischen Kontakt mit der fremden Kultur herstellen können. Podcast im iTunes oder YouTube sind gute Beispiele, mit denen Studenten authentische deutsche Nachrichtenprogramme, TV-programme oder Bücher u.a. erreichen können. Wegen der 
rasanten Entwicklung unserer Welt müssen auch die Deutsch- Curricula verändert und angepasst werden.

Das Ziel der Deutsch-Curricula in Taiwansoll nicht nur in der Ausbildung der sprachlichen Kompetenz vom Lese-, Hör-, Schreib- und Sprech- Verständnis liegen, sondern auch in einem tieferen, der Realität entsprechenden Einblick in Geschichte, Kultur, Politik, Wirtschaft und Gesellschaft der deutschsprachigen Länder. D.h. die Beherrschung der sprachlichen Fertigkeiten ist selbstverständlich wichtig. Was aber zudem nicht zu vernachlässigen ist, ist die Kompetenz von "Literacy“, damit die fremde Kultur angemessen vermittelt wird. Hier wird "Literacy“ im Rahmen der Vermittlung der Interkulturalität erklärt.

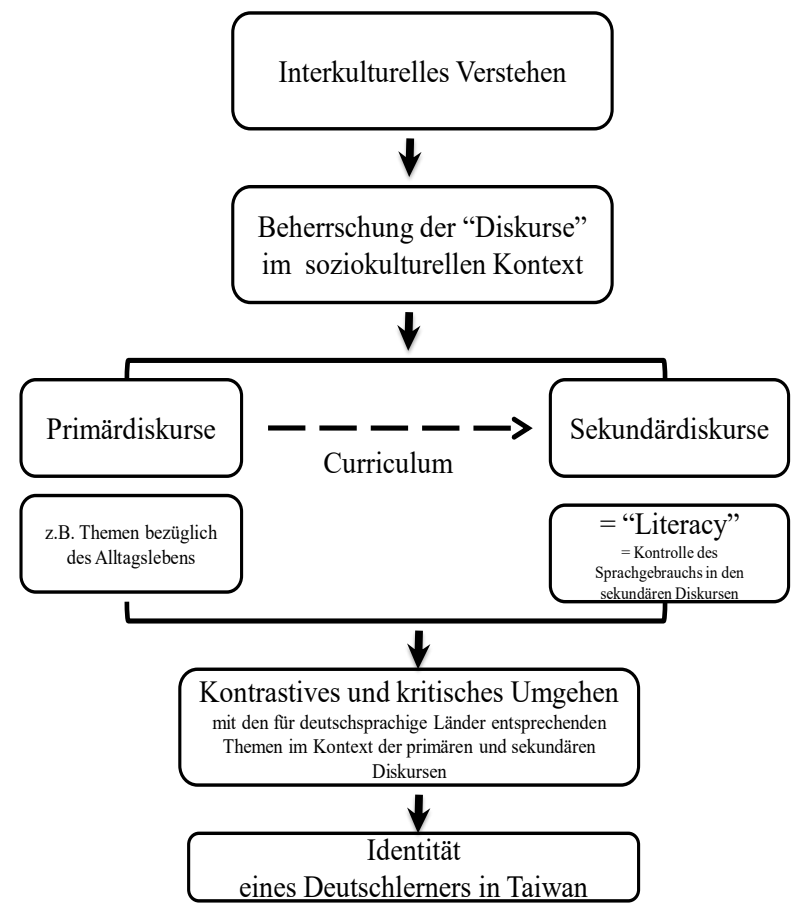

Auf den Begriff „interkulturell“ weist Kramsch hin: dieser werde benutzt, to characterise the acquisition of information about the customs, institutions and history of a society other than one's own." $(1995,87)$ Pädagogisch wird in dieser vorliegenden Arbeit „interkulturelles Verstehen“ benutzt, um eine dialogische Kommunikationsweise hervorzuheben, die auf der Beherrschung der Diskurse im soziokulturellen Kontextbasiert.

Futhark 8 (2013)

Lee, Überlegungen zum pädagogischen, 153-164

ISSN 1886-9300 
Auf einer Seite des Curriculums repräsentieren die Primärdiskurse und auf der anderen Seite die Sekundärdiskurse. Die Beherrschung der Primär- und Sekundärdiskurse ermöglicht das Umgehen sowohl mit den für deutschsprachige Länder entsprechenden Themen als auch für die eigenen relevanten Themen in Taiwan. Darin besteht die Identifizierung eines Deutschlerners in Taiwan

Nach Gee $(1991,6)$ gehören zu Literacy nicht nur Lese- und Schreibkompetenzen, sondern „uses of language in secondary discourses“. Gee definiert „Diskurs“ wie folgt: „a socially accepted association among ways of using language, of thinking, and of acting that can be used to identify oneself as a member of a socially meaningful group or "social network“ (1). In diesem Sinne ist es nicht bloß der Diskurs, sondern es sind vielfältige „Diskurse“, die im sozio-kulturellen Kontext stattfinden.

Wenn die/der Lernende eine Fremdsprache wirklich gut beherrschen kann, heißt es, dass sie/er die Fähigkeit hat, nicht nur Primärdiskurse wie z.B. die des Alltagslebens, sondern auch Sekundärdiskurse wie z.B. das Text- und Kontext- verständnis in vielfältigen Bereichen verstehen und führen zu können. Byrnes et al. weisen darauf hin, dass die Beherrschung von denPrimär- bis zu den Sekundärdiskursen konzeptualisiert werden kann ,as beginning with the oral language use of familiar interactions and gradually moving into public, institutional, and professional settings." $(2006,90)^{2}$ Sekundärdiskurse umfassen aktive Politik, aktuelle Wirtschaft, pulsierende Gesellschaft und neuesten Stand der Wissenschaft, oder gar die hohe Kunst der sprachlichen Abstraktionsfähigkeit. In diesem Sinne ist Literacy mit den Sekundärdiskursen identisch. Die Prinzipien des Curriculums liegen im vorgezeichneten Entwicklungsprozess: mit den grundlegenden Primärdiskursen zu beginnen, und dann schrittweise die weiterführenden Sekundärdiskurse anzufügen, womit ein progressives Lernen erreicht werden soll.

Die Beherrschung der Primär- und Sekundärdiskurse im Zusammenspiel mit kontrastiver Linguistik ermöglicht bzw. sensibilisiert das Umgehen sowohlmit den für deutschsprachige Länder entsprechenden Themen als auch für die eigenen relevanten Themen in Taiwan. Dabei impliziert es, dass "interkulturelles Verstehen" kritisches Denken beinhaltet. Die Identifizierung eines Deutschlerners in Taiwan besteht aus einer durch die Fremdsprache internationalisierte, geänderte Persönlichkeit mit Kenntnissen von Ähnlichkeiten und Unterschieden der vielfältigen Kulturen.

2 Siehe dazu ein konkretes Beispiel von Byrnes et al., S. 94f.

Futhark 8 (2013)

Lee, Überlegungen zum pädagogischen, 153-164

ISSN 1886-9300 


\section{Die Identität eines Deutschlerners in Taiwan: Die Diskurse des Selbst und des Anderen}

Ein Deutsch-Curriculum mit gezielter Literacy-Vermittlung fördert nicht nur Beherrschung der vier Fertigkeiten (Lesen, Hören, Schreiben und Sprechen), sondern auch Kenntnisse und Fähigkeiten, die fremde Kultur und die eigene Kultur mit anpassenden Darstellungsweisen wahrzunehmen also quasi als kultureller Mittler zu agieren.

Das heißt auch, dass der Prozess der Vermittlung als ein dialogischer Prozess gilt. Das Individuum mit Kenntnissen von Ähnlichkeiten und Unterschieden der beiden (Sprach-) Kulturen bewegt sich dazwischen, wo nach Homi Bhabha ein dritter Bereich entwickelt wird, der "does not simply revise or invert the dualities, but revalues the ideological bases of division and difference"3. Die dialogische Auseinandersetzung ist ein steter Prozess des Abbauens und Anbauens beider Kulturen. Eine solche Auseinandersetzung fördert kritische interkulturelle Literacies. In diesem Sinne wird nicht Sprache und Kultur, oder Kultur in der Sprache hervorgehoben, sondern nach Kramsch "language as culture" (1995) oder im Lantolf'schen Sinne "languaculture" (2006) im Kontext einer soziokulturellen Perspektive diskutiert und zur Weiterentwicklung benutzt.

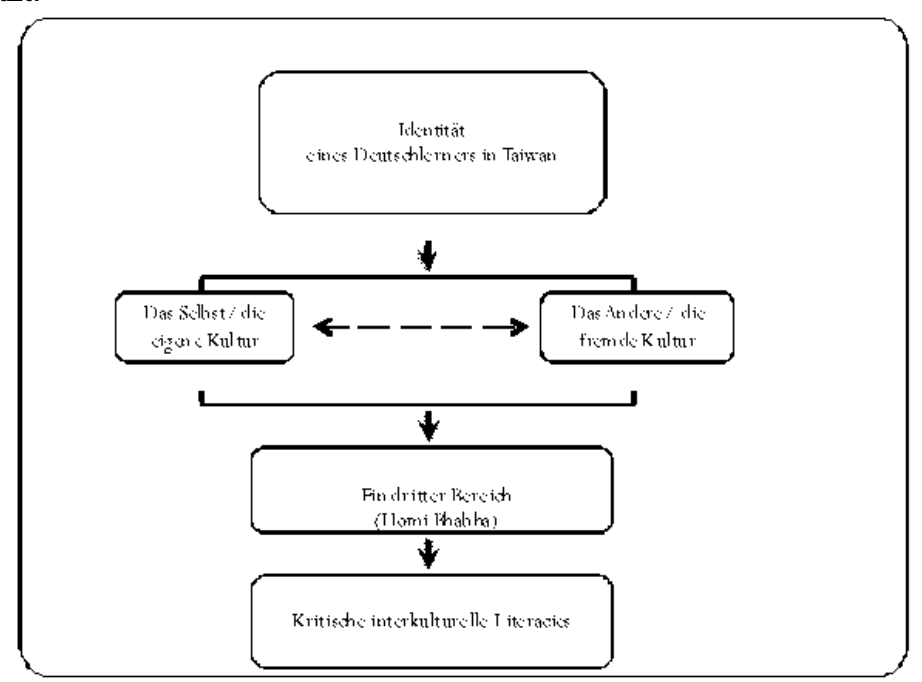

3 Zitiert nach Kramsch, C. (1995), The cultural component of language teaching, S. 89. Zu dem "dritten Bereich" siehe dazu Kramsch, C. (2009), Third culture and language education.

Futhark 8 (2013)

Lee, Überlegungen zum pädagogischen, 153-164

ISSN 1886-9300 
Um „Sprache als Kultur“ im Unterricht zu erzielen, hebt Kramsch kritisch hervor, dass die Fremdsprache als „oppositional practice“ $(1995,90)$ im dritten Bereich unterrichtet werden soll ${ }^{4}$. In diesem Sinne wird nicht das fixierte Lernen des grammatischen, syntaktischen oder semantischen Verständnisses einer Sprache betont, sondern vielmehr die Sprache im sozio-kulturellen Kontext.

\section{Didaktische Überlegung: Interkulturelles Verstehen im Kontext}

Interkulturelles Verstehen bedeutet nicht einfach grammatisches, syntaktisches oder semantisches Verständnis einer Sprache. Die Kenntnis solchen Teilwissens vermittelt noch lange nicht die kulturelle Bedeutung der Worte und Zeichen einer Sprache. Z.B. das Erlernen des Wortes "Gastarbeiter" vermittelt keinerlei Kenntnis über die jeweilige kulturelle Bedeutung/Inhalt dieses Wortes. Ja das Wort könnte sogar missbräuchlich verwendet werden. Aber durch das Lesen eines authentischen Texts z.B. von Dikmen: „Hurra ich lebe in Deutschland oder Die Ergänzung des Märchens" ersteht plötzlich die Kultur im Kontext des Texts ${ }^{5}$. Es geht hier um eine Satire eines türkischen Schriftstellers. Das Leben eines türkischen Gastarbeiters wird nicht unmittelbar beschrieben, sondern durch den Vergleich mit den Deutschen dargestellt. Der Text hilft dem Deutschlerner in Taiwan, im dritten Bereich nicht nur die türkische und die deutsche Kultur „oppositional“ zu lesen, sondern zugleich kritisch und "heteroglossic" (Kramsch 1995, ebd.) mit der eigenen Kultur zu vergleichen. "Kultur" in diesem Sinne ist durch die Darstellungsweise der „Sprache“ verschiedenartig. Die Realisierung der Sprache als Kultur liegt im soziokulturellen Kontext.

4 In Kramschs Text (1995) stellt leider keine didaktische Methode zurV erfügung.

5 Für die Analyse des Texts „Hurra ich lebe in Deutschland oder Die Ergänzung des Märchens" siehe dazu Lee「內容、文體、任務與德語文學教學」(Didaktische Bedeutung von "Inhalt", "Genre" und "Aufgabe" im Literaturunterricht), S. 42-58.

Futhark 8 (2013)

Lee, Überlegungen zum pädagogischen, 153-164

ISSN 1886-9300 


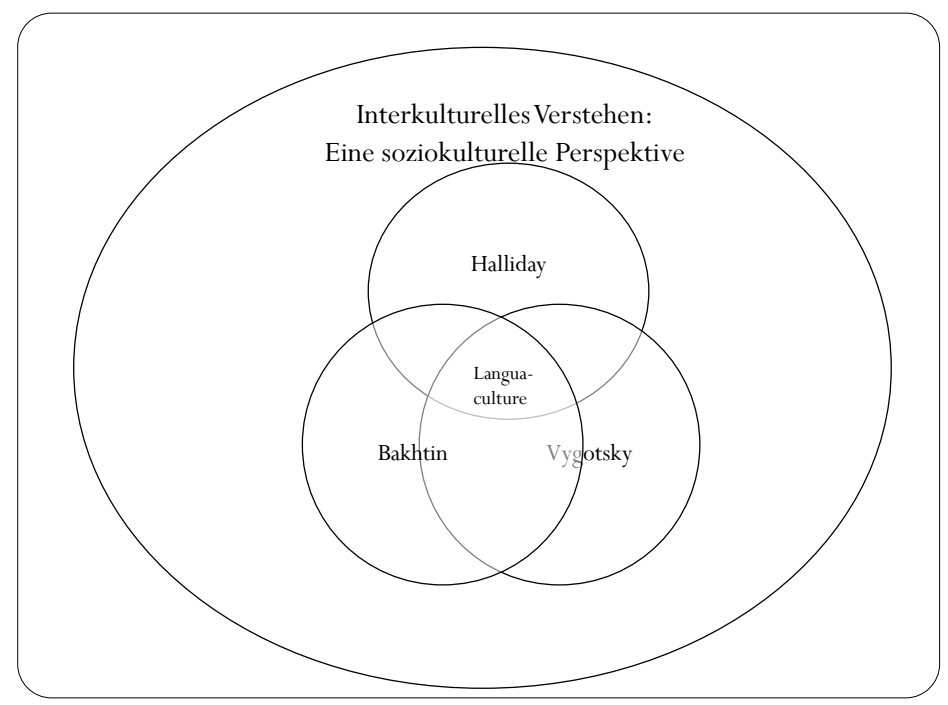

Von der soziokulturellen Perspektive her ist die Sprache bzw. der Signifikant in einer Sprache dynamisch, wird durch die dialogische Interaktion dekonstruiert und neu hergestellt. Nach Vygotsky spielt soziale Interaktion eine entscheidende Rolle für unsere kognitive Entwicklung. Er behauptet: "Any function in the child's cultural development appears twice, or on two planes. First it appears on the social plane, and then on the psychological plane." $(1981,163)$ Die soziale Interaktion bzw. die zwischenpsychologische Interaktion ist beim Fremdsprachen lernen wesentlich, nur dadurch wird die Sprache intrapsychologisch verinnerlicht bzw. das Bewusstsein gegenüber der fremden Kultur entwickelt.

Die soziale Interaktion entspricht Bakhtins These von „Dialog“6. Der Sinn der Sprache ist im Zwischenraum eines Dialogs vom Eigenen und Fremden lokalisiert. Hall hat Bakhtins These wie folgt interpretiert: „meaning is located neither solely in our linguistic resources nor in each individual's mind. Rather, it resides in between these two interdependent spheres, in the interaction, the dialogue, [...]." $(2002,12)$ Um die soziale Interaktion zu ermöglichen, werden die „mediational means“ nach Hall (51) benötigt bzw. es bedarf einer Vermittlungsmethode, die nicht nur linguistisch, sondern auch kulturell im Kontext ist ${ }^{7}$, um zwischen dem

Siehe Bakhtin, The dialogic imagination, S. 293f.

Dies entspricht Hasans Behauptung, dass es eine semiotische und InterBeziehung zwischen der Sprache und den sozialen Aktivitäten gibt. Zitiert nach Byrnes et al., S. 88.

Futhark 8 (2013)

Lee, Überlegungen zum pädagogischen, 153-164

ISSN 1886-9300 
Fremden und dem Eigenen zu kommunizieren und die Welt soziokulturell zu konzeptualisieren.

Zur Analyse der Sprache im Kontext bietet Hallidays Systemische Funktionale Linguistik(1999)eine Methode, bei der die Sprache als soziale Semiotik betrachtet wird ${ }^{8}$.D.h. sein Konzept der Sprache konzentriert sich nicht auf die sprachliche Struktur, sondern auf die Funktion und den Gebrauch der Sprache, spezifisch den Gebrauch der Sprache im soziokulturellen Kontext. In diesem Sinne ist „Kultur“ heterogenisch ${ }^{9} \mathrm{zu}$ verstehen.

Wie Kultur ist die Sprache als soziale Semiotik verschiedenartig und nicht fixiert. Diese Perspektive, wie Kramsch (1995) darstellt, ist gegen die traditionelle Perspektive des Lernens der fixierten Regeln einer Fremdsprache. In jedem Kontext gibt es seine eigenen „Diskurse“, die im Fremdsprachenlernen erworben werden müssen. Z.B. als eine Literaturwissenschaftlerin muss ich den Diskurs in diesem Bereich gut beherrschen, damit ich mit anderen angemessen kommunizieren kann. Der Diskurs beinhaltet Kultur. Der angemessene Diskurs in einem Kontext ist das "Genre", das nach Bakhtin zwei Formen einbezieht: gesprochenes und geschriebenes Genre. Jede Form beinhaltet „staged, goal-oriented purposeful activity with its own schematic structures“" (Martin, 1985, 251). Die Beherrschung des jeweiligen Genres verdeutlicht bei der sozialen Interaktion das interkulturelle Verstehen.

Zusammenfassend heben Vygotsky, Bakhtin und Halliday gemeinsam hervor, dass eine Sprache im soziokulturellen Kontextdynamisch ist. Das Lernen einer Fremdsprache ist das Erlernen der sozialen Interaktion in einer dynamischen Weise. In diesem Sinne ist die Sprache die Kultur, die im Deutschunterricht insbesondere durch einen authentischen Text im Kontext vermittelt wird.

\section{Zur Vermittlung der Kultur: Die Genre- und Handlung- orientierte Kompetenz}

Beim Fremdsprachenlernen ist es für Anfänger nötig, die fixierte Grammatik zu lernen. Es bleibt dann fragwürdig, wie man das Fremde gut kennenlernen kann, dazu das Eigene ergänzt, und im dritten Bereich dynamisch einen Kontext inszeniert. Byrnes et al. (2006) ${ }^{10}$ als Pioniere für die neue Konstruktion des Deutsch-Curriculums betten Genre und

8 Vgl. Eggins ausführliche Einleitung in: An introduction to systemic functional linguistics.

9 Siehe dazu Kramsch, S. 89

10 Siehe dazu Byrnes theoretische Überlegung zur Curriculumkonstruktion in "What kind of resource is language and why does it matter for advanced language learning? An introduction". 
Handlung ein, um zwischen dem fixierten Lernen und der Dynamik der sozialen Interaktion die Flexibilität zu finden.

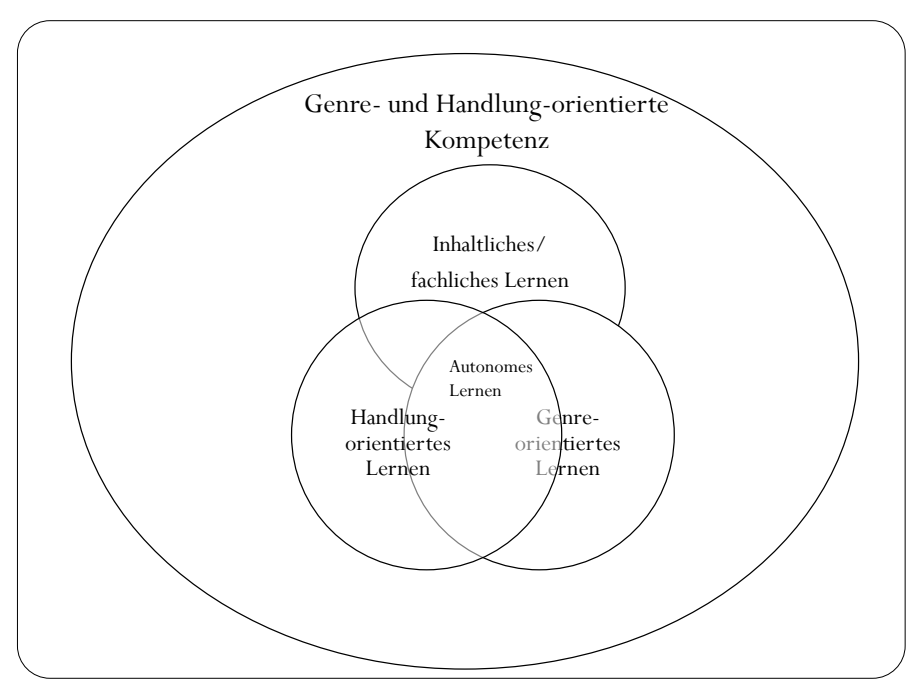

Die von Byrnes et al. dargestellte Genre-orientierte Handlung als Aufgabe ergänzt konkret die fehlende pädagogische Überlegung in Kramschs Arbeit (1995) und ermöglicht die Sprache-als-Kultur-Vermittlung. Ein solches Lernen verwirklicht eine authentische Kommunikationsweise im Unterricht mit verschiedenen Genres und Inhalten. Ein Beispiel des inhaltliches Lernens nach Lee $(2010,47)$ mit der sprachlichen und kulturellen Vermittlungsweise ist wie folgt:

\begin{tabular}{||c||c||}
\hline Thema & Text \\
\hline \hline "Deutschland“ & 1. Thomas „Das Salz auf der Pizza“ \\
Aus eigener & 2. Kaminer „Junggesellen und Familien- \\
Sicht & wirtschaft“ \\
& 3. Oswald, „Alles was zählt“ \\
\hline \hline "Deutschland“ & Dikmen, „Hurra ich lebe in Deutschland \\
aus ausländi- & oder Die \\
scher Sicht & Ergänzung des Märchens“ \\
\hline \hline
\end{tabular}

Futhark 8 (2013)

Lee, Überlegungen zum pädagogischen, 153-164

ISSN 1886-9300 
Die ausgewählten Themen sind im Rahmen eines Deutschcurriculums für die fortgeschrittenen Lerner mit Deutsch als Hauptfach in Taiwan ${ }^{11}$. Nach dem Lesen von Dikmens Satire werden die Studenten aufgefordert, eine eigene Satire zu schaffen. Das Thema ist nicht Gastarbeiter in Deutschland, sondern Gastarbeiter in Taiwan, um damit die empfundenen kulturellen Unterschiede und Ähnlichkeiten aufzuzeigen. Um diese geschriebene Aufgabe zu schaffen, müssen die Studenten auf die inhaltliche und sprachliche Ebene achten. Auf der sprachlichen Ebene sind die semantischen, syntaktischen, und diskursiven Aspekte eingeschlossen. Auf der inhaltlichen Ebene müssen die Studenten die Kenntnisse von Dikmens Text einbeziehen, kritisch bzw. satirisch über die Situation der Gastarbeiter in Taiwan nachdenken, und ein satirisches Genre entsprechend ihrer Meinung erarbeiten. Die sprachliche Ebene gehört zu dem nach Byrnes et al. (2006) fixierten Lernen, während die inhaltliche Ebene vielmehr dynamisch ist. Die beiden helfen dem Lernenden, die eigene Meinung dem Genre entsprechend auszudrücken.

Die Zielsetzung des aufgabenorientierten Lernens ist das Bewältigen sinnvoller kommunikativer Aufgaben, mit welchen die Lerner im wirklichen Leben bzw. in den deutschsprachigen Ländern konfrontiert werden. Aktivitäten dieser Art fördern kritisches Nachdenken, und den selbstgesteuerten Kenntniserwerb. Die interkulturelle Lernerautonomie bedeutet in diesem Sinne '(de)konstruktivistisches Lernen' mit derlei explorierenden kulturellen Themen im Unterricht, d.h. für das weitere Lernen haben die Lerner die Fähigkeit, im dritten Bereich zustehen und die Kulturen kritisch bzw. kreativ zu vermitteln.

Der Genre- und Aufgabe-orientierte Unterricht wird mit lehrergesteuerter Fokussierung auf der konkreten sprachlichen Ebene verbunden und kann die Flüssigkeit und Sprachlernfertigkeit der Lerner verbes$\operatorname{sern}^{12}$.

\section{Schlussbemerkung}

Zusammenfassend lässt sich sagen, dass interkulturelles Verstehen in diesem Kontext einen soziokulturellen Dialog mit angemessenen Diskursen bedeutet. Der Lerner ist fähig, mittels der Fremdsprache nicht nur die fremde Kultur zu verstehen, sondern das eigene Selbst mit einer

11 Andere aus gewählteThemen für den Lesekurs sind beispielsweis eim Rahmen von Politik, Wirtschaft, Erziehung, Psychologie und Umweltschutz. Vgl. dazu Byrnes Deutschcurriculumdesign an der Georgetown Universität und das im Text "Taking text to task: Issues and choices in curriculum construction".

12 Über die Genre-orientierte Handlung, vgl. auch Yasudas Arbeit "Genre-based tasks in foreign language writing: Developing writers' genre awareness, linguistic knowledge, and writing competence". 
angemessenen Meinung darzustellen. Der Einbau von „Literacy“ in das Curriculum zielt nicht nur auf Sekundärdiskurse ab, wie das Gee gesagt hat. Es sind Literacies der vielfältigsten Bereiche mit verschiedenen Genres, die auf die Identität eines Deutschlerners in Taiwan Einfluss ausüben. Wir hoffen, dass der Student nicht nur die Fremdsprache perfektioniert, sondern auch ein kritisch-kreativer Mittler im raschen Wandel der Kulturen wird.

\section{Zitierte Literatur:}

BAKHTIN, M.M. (1981): The dialogic imagination. Austin, University of Texas.

Byrnes, H. (2006): What kind of resource is language and why does it matter for advanced language learning? An introduction. In: $\mathrm{H}$. Byrnes ( $\mathrm{Hg}$.), Advanced language learning: The contribution of Halliday and Vygotsky, (S. 1-28), London, Continuum.

Byrnes, H., Crane, C., Maxim, H. H., \& Sprang, K. A. (2006): Taking text to task: Issues and choices in curriculum construction. In: International Journal of Applied Linguistics 152, (S. 85-110), Blackwell, New Jersey.

EgGINS, S. (2004): An introduction to systemic functional linguistics. 2Aufl. London, Continuum.

GEE, J. P. (1991): What is literacy? In: C. Mitchell and K. Weiler (Hg.), Rewriting Literacy: Culture and the Discourse of the Other, (S. 1-11), New York, Bergin and Garvey.

HALL, J. K. (2002): Teaching and Researching Language and Culture. London, Pearson Education.

HALLIDAY, M.A.K. (1999): The notion of "context" in language education. In: M.Ghadessy (Hg.), Text and context in functional linguistics, (S.1-24), Amsterdam/Philadelphia, John Benjamins.

KRAMSCH, C. (1995): The cultural component of language teaching. In: Language, culture and curriculum, 8 (2), (S. 83-92), London \& New York, Routledge.

---. (2009): Third culture and language education. In: Vivian Cook \& Li Wei (Hg.), Contemporary Applied Linguistics. Vol.1, Language Teaching and Learning, (S.233-254), London, Continuum.

Lantolf, J. (2006): Re(de)fining language proficiency in light of the concept of ,languaculture'. In: H. Byrnes (Hg.), Advanced language learning: The contribution of Halliday and Vygotsky, (S. 72-91), London, Continuum.

LEE, SP.(2010): Didaktische Bedeutung von „Inhalt“, „Genre“ und „Aufgabe“ im Literaturunterricht ( $「$ 內容」、「文體」、「任務」與德語文學教學). In: Lernen und Lehren: Deutsche Sprache, Literatur und Kultur, (S. 42-58),Taipei, Kuantang.

MARTIN, J. (1985): Process and text: two aspects of human semiosis. In: J. D. BENSON \& W. S. GREAVES (Hg.), Systemic perspectives on discourse: selected theoretical papers from the $9^{\text {th }}$ International systemic workshop, (S. 248274), Norwood, NJ: Ablex.

Futhark 8 (2013)

Lee, Überlegungen zum pädagogischen, 153-164

ISSN 1886-9300 
Vygotsky, L. S. (1981): The genesis of higher mental functions. In: J.V. Wertsch (Hg. und Übers.), The concept of activity in Soviet psychology. Armonk, NY, MIT Press.

Yasuda, S. (2011): Genre-based tasks in foreign language writing: Developing writers' genre awareness, linguistic knowledge, and writing competence. In: Journal of second language writing 20, (S. 111-33), Netherlands, Elsevier. 